\title{
Lower Limb Ulcer Associated with Peritonitis in Patients Undergoing Peritoneal Dialysis
}

This article was published in the following Dove Press journal:

International Journal of Nephrology and Renovascular Disease

\section{Kohsuke Terada \\ Sae Aratani \\ Akio Hirama \\ Tetsuya Kashiwagi (D) \\ Yukinao Sakai (iD}

Department of Nephrology, Graduate School of Medicine, Nippon Medical School, Tokyo, Japan
Correspondence: Yukinao Sakai Department of Nephrology, Graduate School of Medicine, Nippon Medical School, I-I-5 Sendagi, Bunkyo-Ku, Tokyo, II 3-8603, Japan

Tel +8I-3-3822-2I3I ext. 6496

Fax +8I-3-3822-4865

Email y-sakai@nms.ac.jp
Purpose: Peritoneal dialysis (PD)-related peritonitis and lower limb ulcer are the important complications in patients undergoing PD. Although the association between lower limb ulcer and peritonitis in patients undergoing PD is unclear, based on our clinical experience and the clinical importance of the complications in patients undergoing PD, we hypothesized that lower limb ulcer is associated with peritonitis in patients on PD.

Patients and Methods: In this single center, retrospective cohort study, we studied 87 patients who started undergoing PD at our hospital from April 2015 to March 2020. We compared these 8 patients with lower limb ulcer with the other 79 patients without lower limb ulcer. We compared between the patients in the objection period of this study about peritonitis using Log rank test, and used the unpaired $t$-test and Fisher's exact test to compare the clinical factors between the two groups. Moreover, we used univariate and multivariate logistic regression analyses to study the association of PD-related peritonitis with the clinical factors.

Results: The period developed first peritonitis of the patients on PD with lower limb ulcer was significant shorter than those without lower limb ulcer in Log rank test $(P=0.011)$. The Fisher's exact test and unpaired $t$-test showed that the difference in the prevalence of PDrelated peritonitis $(\mathrm{P}=0.009)$, peritonitis/patient years $(\mathrm{P}=0.036)$, the $\mathrm{BMI}(\mathrm{P}=0.007)$ and icodextrin $(\mathrm{P}=0.001)$ were significant. Lower limb ulcer had significant associations with peritonitis in patients on $\mathrm{PD}$ in both univariate [odds ratio (OR) 8.461, 95\% confidence interval (CI) $1.854-45.60, \mathrm{P}=0.006$ ] and multivariate [OR 7.169, 95\% CI 1.519-39.480, $\mathrm{P}=$ $0.013]$ logistic regression analysis.

Conclusion: In conclusion, lower limb ulcer may be associated with peritonitis in patients undergoing PD. Further large-scale, prospective studies are required to confirm these results. Keywords: peritoneal dialysis, peritonitis, lower limb ulcer

\section{Introduction}

Peritoneal dialysis (PD)-related peritonitis is one of the most common and important complications in patients undergoing $\mathrm{PD}$ that requires hospitalization and/or transfer to permanent hemodialysis and may cause death. ${ }^{1-3}$ Therefore, the prediction and prevention of peritonitis in patients undergoing PD are essential for improving the quality of life in these patients. In 2016, the International Society for Peritoneal Dialysis (ISPD) published the guidelines entitled, "ISPD Peritonitis Recommendations: 2016 Update on Prevention and Treatment," for peritonitis in patients undergoing PD. ${ }^{1}$ Awareness and information regarding the risk factors for peritonitis in PD patients are important to prevent PD-related peritonitis; several risk factors for PD-related peritonitis, such as diabetes mellitus (DM) and smoking, have been listed in the guidelines and in other reports. ${ }^{1-11}$ We have used the 
protocols and information in these guidelines to manage peritonitis in PD patients at our hospital. Nevertheless, in PD patients, peritonitis remains a severe problem, which requires to be addressed.

The most common primary cause of end-stage renal disease is diabetic nephropathy that results from poorly controlled DM in the United States of America and Asia, including Japan. ${ }^{12-14}$ Furthermore, DM is a risk factor for peritonitis $^{1,2,5-7,9,10}$ and lower limb ulcer in patients undergoing dialysis. ${ }^{15}$ In addition, PD patients with DM are at a greater risk of lower extremity amputation than those without DM. ${ }^{16}$ Based on our experience, we can state that peritonitis may be associated with lower limb ulcer in patients undergoing PD, irrespective of the present of DM. However, to the best of our knowledge; no study has reported an association of lower limb ulcer with peritonitis in patients on PD, and the association between lower limb ulcer and peritonitis in patients undergoing PD is unclear.

We hypothesized that lower limb ulcer is associated with peritonitis in patients on PD, because both the lower limb ulcer and peritonitis are very important factors in patients on PD and the two factors may have the mutual problems such as the infection. Therefore, we designed this study to investigate whether lower limb ulcer is associated with peritonitis in patients undergoing PD.

\section{Patients and Methods}

\section{Study Population and Study Design}

In this single center, retrospective cohort study, we enrolled patients who provided informed consent for study participation, with the option of opting out. Those who refused to participate in the trial were not enrolled. The study protocol was approved by the Ethics Committee of the Nippon Medical School Hospital (B-2020-130) and registered at the University Hospital Medical Information Network as UMIN No. 000046719. The study was performed in keeping with the principles in the Helsinki Declaration.

We studied 87 patients who started undergoing PD at our hospital, the Nippon Medical School Hospital, and attended our hospital regularly from April 2015 to March 2020; those on temporary PD were not enrolled in our trial. We defined that we followed up the patients until PD cessation by all causes including death and the end of the study period in this study. We defined the patients undergoing combination therapy with hemodialysis as the patients undergoing PD. We collected data regarding two clinical factors, lower limb ulcer and peritonitis, from the study subjects. Of the 87 study subjects, eight patients had experienced episodes of lower limb ulcer. Therefore, in this study, we compared these eight patients with lower limb ulcer with the other 79 patients without lower limb ulcer. Peritonitis was present in 18 patients: 5 from the patients with lower limb ulcer and 13 from the patients without lower limb ulcer. Figure 1 shows the flowchart of the study design and subject enrollment.

\section{Definitions of the Terms in This Study}

In this study, we defined lower limb ulcer as the presence of a single ulcer or multiple ulcers. The lower limb ulcers that needed treatment were evaluated and characterized at

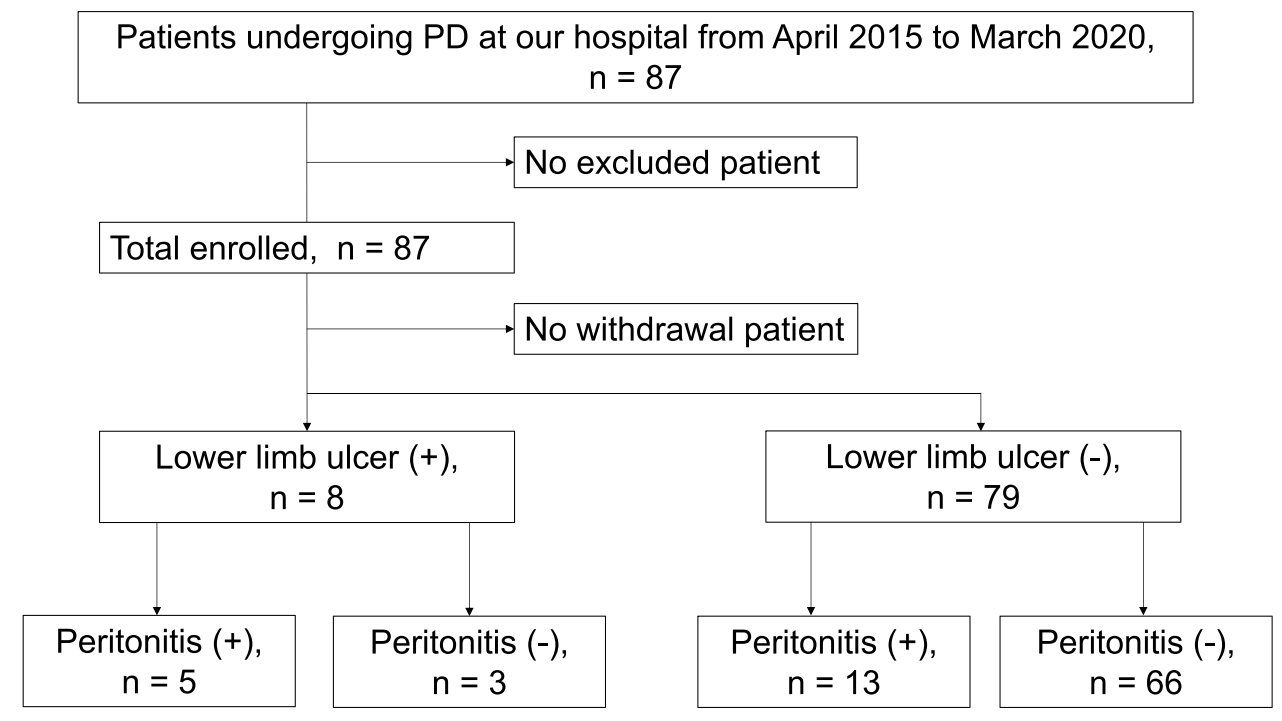

Figure I Flowchart of the study design. Abbreviation; PD, peritoneal dialysis. 
the department of plastic surgery or dermatology department at our hospital. We did not perform detailed classification of the types of lower limb ulcers owing to the relatively small number of subjects who had lower limb ulcer.

We defined the patients with malignant disease as those with malignant disease during or after the initiation of PD. We did not classify the subjects as per the type of malignant disease owing to the limited number of subjects.

An ankle-brachial index $(\mathrm{ABI})<0.9$ at the time of PD initiation was considered to be abnormal ABI.

\section{The Survival Analysis Between the Patients with and without Lower Limb Ulcer by Log Rank Test}

We compared between the patients with and without lower limb ulcer in the objection period of this study about peritonitis using Log rank test to investigate the association between PD-related peritonitis and lower limb ulcer in patients undergoing PD; we defined the patients had lower limb ulcer occurred by the end of the period of this study in this study as the patients with lower limb ulcer, and we defined the endpoint as the first PD related peritonitis in this Log rank test.

\section{A Comparison of Patients with and without Lower Limb Ulcer Using Unpaired t-Test and Fisher's Exact Test \\ Analysis}

We used the unpaired $t$-test and Fisher's exact test to compare the following clinical factors between the 8 patients with lower limb ulcer and the 79 patients without lower limb ulcer: sex, age, body mass index (BMI), presence of DM, nephrosclerosis as the primary disease, estimated glomerular filtration rate at PD initiation, dialysate creatinine to plasma creatinine ratio $(\mathrm{D} /$ $P$ ratio), dialysate glucose to initial dialysate glucose ratio (D/D ratio), hypertension, current and former smoking, presence of malignant disease, cardiovascular disease at PD initiation, diverticulosis, arrhythmia, abnormal ABI, exit-site infection, PD-related peritonitis, hemoglobin $(\mathrm{Hb})$ level at PD initiation, serum potassium $(\mathrm{K})$ level at $\mathrm{PD}$ initiation, serum albumin (Alb) at PD initiation, and C-reactive protein (CRP) level at PD initiation, automated PD, combination therapy with hemodialysis, icodextrin, follow-up period, and peritonitis/patients years. All patients used the automated connecting and disconnecting device with ultraviolet light on PD.

\section{The Association Between Lower Limb Ulcer and PD-Related Peritonitis}

We investigated the association between PD-related peritonitis and lower limb ulcer in the study subjects. We used univariate and multivariate logistic regression analyses to study the association of PD-related peritonitis with ten clinical factors (lower limb ulcer, exit-site infection in PD, presence of DM, abnormal status of ABI, cardiovascular disease at PD initiation, arrhythmia, diverticulosis, current and former smoking, icodextrin, and BMI) because the sample size was not sufficient to allow robust statistical analyses, and we considered the clinical importance of the factors in this study about PD-related peritonitis and lower limb ulcer. Moreover, we attempted to identify the bacteria responsible for peritonitis and lower limb ulcer in the eight patients with lower limb ulcer.

\section{Statistical Analyses}

All the laboratory values have been presented as mean \pm standard deviation values. Continuous variables were compared with the unpaired $t$-test, and survival analysis was performed on longitudinal data to address its multiplicity. Fisher's exact test was used for various inter-group comparisons. The Mantel-Cox Log rank test was used to compare survival curves. P values $<0.05$ were considered to indicate statistical significance. All the statistical analyses were performed using Prism $^{\circledR}$ software version 8 (GraphPad Software, La Jolla, CA, USA). Furthermore, logistic regression analyses were performed using $\mathrm{JMP}^{\circledR}$ software version 14 (SAS Institute, Cary, NC, USA).

\section{Results}

\section{The Survival Analysis Between the Patients with and without Lower Limb Ulcer by Log Rank Test}

The period developed first peritonitis of the patients on PD with lower limb ulcer was significant shorter than the period of the patients on PD without lower limb ulcer in Log rank test $(P=0.011)$. Figure 2 shows the results.

\section{Basic Characteristics of Participants and the Comparison of Patients with and without Lower Limb Ulcer Using Unpaired $t$-Test and Fisher's Exact Test}

The study enrolled 87 PD patients (65 men and 22 women). As a result, there were no excluded patients and withdrawal patients in this study. The most common 


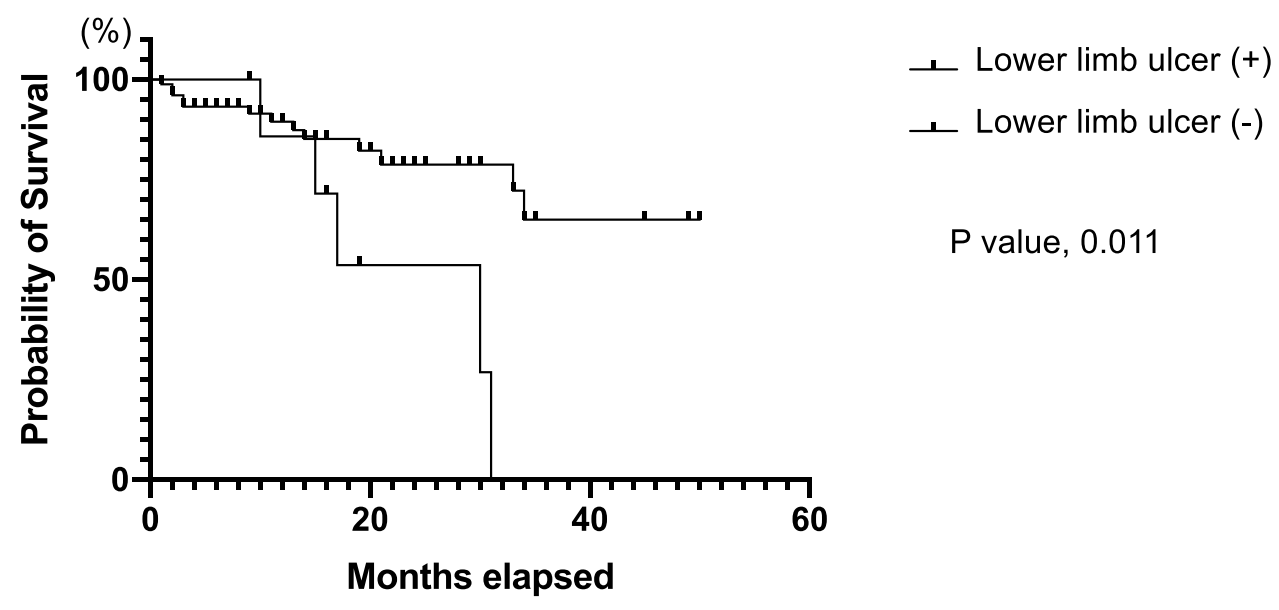

Figure 2 The survival analysis between the patients with and without lower limb ulcer.

complication was hypertension (83 patients, 95.4\%), followed by DM (51 patients, $58.6 \%$ ). The Fisher's exact test showed that the difference in the prevalence of PD-related peritonitis was significant between patients with and without lower limb ulcer $(P=0.009)$. Moreover, the Fisher's test showed that the difference in peritonitis/patient years was significant between patients with and without lower limb ulcer $(\mathrm{P}=0.036)$.

Although this was not the primary aim of our study, the BMI $(P=0.007)$ and icodextrin $(P=0.001)$ were also significantly different between the groups.

Table 1 shows the basic characteristics of the participants and the results of the unpaired $t$-test and Fisher's test analysis for patients on PD with and without lower limb ulcer in this study.

\section{Lower Limb Ulcer, Peritonitis, and DM in the Study Subjects}

In this study, 18 patients developed peritonitis. Further, eight patients had the episode of lower limb ulcer in this study, and five of them developed peritonitis. Six of the eight patients with lower limb ulcer had DM; however, one of the five patients who developed peritonitis after lower limb ulcer did not have DM. Of the five patients who developed peritonitis after lower limb ulcer, we detected the same organ from cultures of lower limb ulcer and effluent at peritonitis in only one patient. Table 2 shows the details of patients who had lower limb ulcer during the study period with and without peritonitis.

\section{The Association Between Lower Limb Ulcer and PD-Related Peritonitis}

As per univariate logistic regression analysis, lower limb ulcer [odds ratio (OR) 8.461, 95\% confidence interval (CI)
1.854-45.60, $\mathrm{P}=0.006]$ had a significant association with peritonitis in patients on PD. Other factors, such as abnormal $\mathrm{ABI}$ and presence of DM, did not show significant associations with peritonitis in patients on PD. Moreover, lower limb ulcer [OR 7.169, 95\% CI 1.519-39.480, $\mathrm{P}=0.013$ ] had a significant association with peritonitis in patients undergoing PD on multivariate logistic regression analysis; we adopted lower limb ulcer and presence of DM as independent variables in multivariate logistic regression analysis, because the sample size was not sufficient to allow robust statistical analyses and we thought that the two factors had clinical importance in this study about lower limb ulcer and peritonitis; we thought presence of DM was considered as the most important confounding factor. In addition, BMI and icodextrin that had significant differences between the patients with and without lower limb ulcer in Fisher's exact test and unpaired $t$-test analysis were not significant in the univariate logistic regression analysis.

In this study, lower limb ulcer had a significant association with peritonitis in PD patients as per the univariate and multivariate logistic regression models. Table 3 shows the results of the univariate and multivariate logistic regression analyses.

\section{Discussion}

In patients undergoing PD, PD-related peritonitis is one of the most common and important complications; peritonitis is associated with hospitalization, transfer to permanent hemodialysis, and death in these patients. ${ }^{1,2,3}$ Therefore, it is crucial to prevent the occurrence of peritonitis in patients on $\mathrm{PD}$; this indicates the importance of accurate prediction of peritonitis that would enable its prevention. Several studies have reported on PD-related peritonitis, 
Table I Baseline Characteristics and Parameters of the Patients

\begin{tabular}{|c|c|c|c|c|}
\hline \multicolumn{5}{|c|}{ Baseline Characteristics and Parameters of Patients } \\
\hline \multirow[t]{2}{*}{ Total, $\mathbf{n}$} & All Patients & Patients with Lower Limb Ulcer & Patients without Lower Limb Ulcer & \multirow[t]{2}{*}{$P$ value* } \\
\hline & $\mathbf{n}=\mathbf{8 7}$ & $\mathrm{n}=\mathbf{8}$ & $n=79$ & \\
\hline Female, $\mathrm{n}(\%)$ & $22(25.3)$ & $\mathrm{I}(12.5)$ & $21(26.6)$ & 0.674 \\
\hline Age (years) & $62.31 \pm 14.55$ & $60.75 \pm 12.20$ & $62.47 \pm 14.82$ & 0.752 \\
\hline BMI $\left(\mathrm{kg} / \mathrm{m}^{2}\right)$ & $23.99 \pm 3.86$ & $27.42 \pm 5.35$ & $23.62 \pm 3.53$ & 0.007 \\
\hline Diabetes mellitus, $\mathrm{n}(\%)$ & $51(58.6)$ & $6(75.0)$ & $45(57.0)$ & 0.461 \\
\hline Nephrosclerosis as primarily disease, $n(\%)$ & $22(25.3)$ & $2(25.0)$ & $20(25.3)$ & $>0.999$ \\
\hline eGFR at PD initiation $\left(\mathrm{mL} / \mathrm{min} / 1.73 \mathrm{~m}^{\wedge} 2\right)$ & $7.167 \pm 3.449$ & $6.188 \pm 1.462$ & $7.266 \pm 3.580$ & 0.403 \\
\hline $\mathrm{D} / \mathrm{P}$ ratio & $0.680 \pm 0.148$ & $0.646 \pm 0.110$ & $0.685 \pm 0.152$ & 0.485 \\
\hline D/D0 ratio & $0.383 \pm 0.099$ & $0.416 \pm 0.095$ & $0.379 \pm 0.099$ & 0.318 \\
\hline Hypertension, $n$ (\%) & $83(95.4)$ & $8(100)$ & $75(94.9)$ & $>0.999$ \\
\hline Smoking, n (\%) & $52(59.8)$ & $4(50.0)$ & $48(60.8)$ & 0.608 \\
\hline Malignant disease, $\mathrm{n}(\%)$ & $16(18.4)$ & $0(0)$ & $16(20.3)$ & 0.342 \\
\hline CVD at PD initiation, $n(\%)$ & $24(27.6)$ & $3(37.5)$ & $21(26.6)$ & 0.679 \\
\hline Diverticulosis, n (\%) & $34(39.0)$ & $5(62.5)$ & $29(36.7)$ & 0.255 \\
\hline Arrhythmia, n (\%) & $9(10.3)$ & $\mathrm{I}(12.5)$ & $8(10.1)$ & $>0.999$ \\
\hline Abnormal status of $A B I, n(\%)$ & $5(5.7)$ & $0(0)$ & $5(6.33)$ & $>0.999$ \\
\hline Exit-site infection, n (\%) & $14(16.1)$ & $\mathrm{I}(12.5)$ & $13(16.5)$ & $>0.999$ \\
\hline $\mathrm{Hb}$ at $\mathrm{PD}$ initiation $(\mathrm{g} / \mathrm{dL})$ & $9.973 \pm 1.156$ & $9.450 \pm 1.294$ & $10.027 \pm 1.136$ & 0.180 \\
\hline Serum $K$ at $P D$ initiation $(m E q / L)$ & $4.519 \pm 0.642$ & $4.450 \pm 0.469$ & $4.526 \pm 0.657$ & 0.753 \\
\hline Serum Alb at PD initiation $(g / d L)$ & $3.355 \pm 0.617$ & $2.950 \pm 0.709$ & $3.396 \pm 0.596$ & 0.051 \\
\hline CRP at PD initiation (mg/dL) & $0.470 \pm 0.869$ & $0.318 \pm 0.329$ & $0.486 \pm 0.907$ & 0.605 \\
\hline Automated PD, n (\%) & $53(60.9)$ & $4(50.0)$ & $49(62.0)$ & 0.706 \\
\hline Combination therapy with hemodialysis, $n$ (\%) & II (12.6) & $2(25.0)$ & $9(11.4)$ & 0.266 \\
\hline Icodextrin, n (\%) & $39(44.8)$ & $8(100)$ & $31(39.2)$ & 0.001 \\
\hline PD-related peritonitis, n (\%) & $18(20.7)$ & $5(62.5)$ & $13(4.35)$ & 0.009 \\
\hline Follow-up period (months) & $19.287 \pm|2.50|$ & $22.125 \pm 8.774$ & $19.000 \pm 12.825$ & 0.139 \\
\hline Peritonitis/patient years & 0.129 & 0.339 & 0.104 & 0.036 \\
\hline
\end{tabular}

Notes; Plus-minus values are means \pm standard division. $* \mathrm{P}$ values are for the comparisons between patients with and without lower limb ulcer.

Abbreviations; BMI, body mass index; eGFR, estimated glomerular filtration rate; PD, peritoneal dialysis; D/P ratio, dialysate creatinine to plasma creatinine ratio; D/D ratio, dialysate glucose to initial dialysate glucose ratio; CVD, cardiovascular disease; $\mathrm{ABI}$, ankle-brachial pressure index; Hb, hemoglobin; $\mathrm{K}$, serum potassium; Alb, albumin; CRP, C-reactive protein.

and various risk factors, such as DM and smoking, have been implicated in peritonitis among patients on PD. ${ }^{1-11}$ The ISPD has published guidelines for PD-related peritonitis. ${ }^{1}$ In our hospital, we manage patients on PD with peritonitis as per these guidelines. However, peritonitis remains one of the most severe complications and an important problem in patients undergoing $\mathrm{PD}$, which needs to be resolved.

Lower limb ulcer is reportedly associated with lower extremity amputation and mortality. ${ }^{17}$ Peripheral artery disease is associated with mortality in patients with chronic kidney disease. ${ }^{18}$ Patients undergoing dialysis are at a higher risk of lower limb complications and ulcer associated with lower extremity amputation and mortality. ${ }^{17,19,20}$

As previously shown, several reports have demonstrated that DM, a common complication in patients undergoing $\mathrm{PD}$, is a risk factor for PD-related peritonitis. $1,2,5-7,9,10$ Furthermore, patients with DM undergoing dialysis are at a higher risk of lower limb ulcer than those who do not have DM. ${ }^{15}$ Diabetic foot ulcers are associated with a high rate of lower extremity amputation, and the 5-year mortality in patients with diabetic foot disease is high, approaching $50 \% .^{21,22}$ The 1-year mortality in DM patients on dialysis who had to undergo lower extremity amputation is reported to be $50.8 \%{ }^{23}$ Patients on PD with DM are at a higher risk of lower extremity amputation than those without DM, and regular monitoring of the feet is very important for patients on $\mathrm{PD}$ with DM. ${ }^{16,24}$ Considering these reports, lower limb diseases are important complications in patients undergoing PD.

Both PD-related peritonitis and lower limb ulcer are common and important complications in patients on PD. However, to the best of our knowledge, no report has discussed the association of lower limb ulcer and PDrelated peritonitis in patients undergoing PD. 


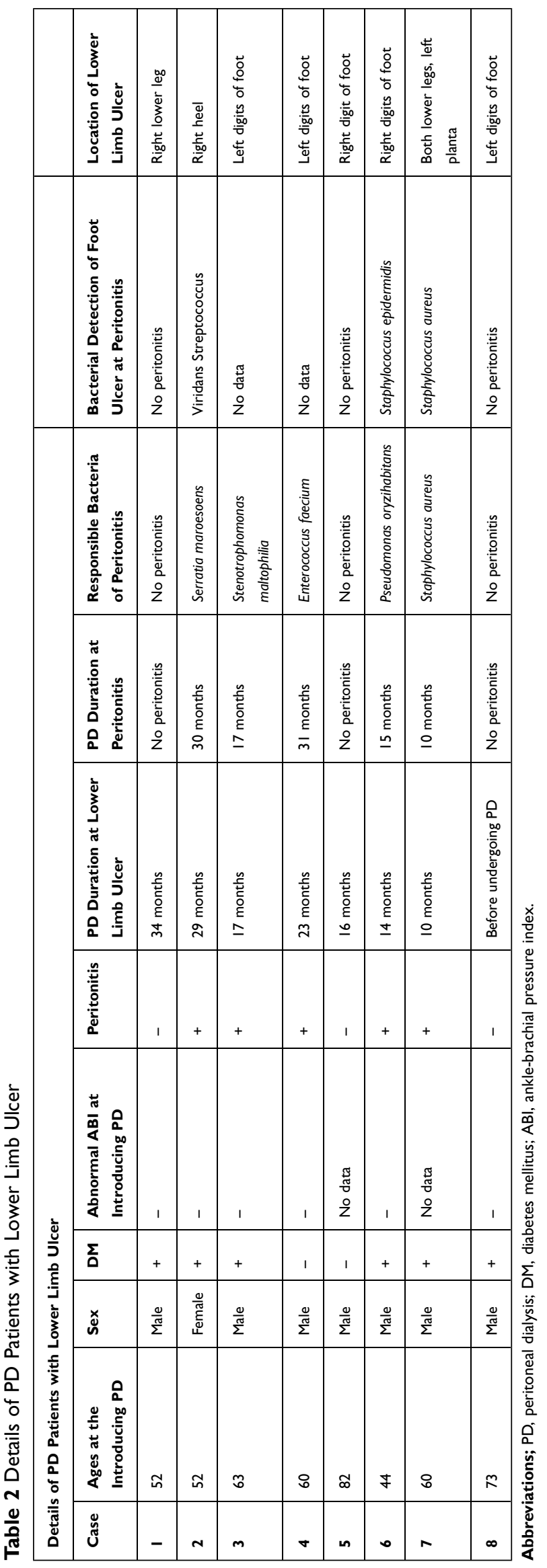

Based on our clinical experience and the clinical importance of both PD-related peritonitis and lower limb ulcer when we manage the patients on PD, we hypothesized that the occurrence of lower limb ulcer is associated with peritonitis in patients undergoing PD.

As the result, the period developed first peritonitis of patients with lower limb ulcer was significant shorter than the period of patients without lower limb ulcer in Log rank test. In addition, we compared the basic characteristics of participants with and without lower limb ulcer using unpaired $t$-test and Fisher's exact test analysis. In this study, the prevalence of PD-related peritonitis in patients on PD showed a significant difference in Fisher's exact test analysis between patients with and without lower limb ulcer. Moreover, the Fisher's test showed that the difference in peritonitis/patient years was significant between patients with and without lower limb ulcer. Moreover, we investigate the association peritonitis with the ten factors such as lower limb ulcer in univariate and multivariate logistic regression models; we included icodextrin and BMI that had significant differences between the patients with and without lower limb ulcer in Fisher's exact test and unpaired $t$-test analysis in univariate logistic regression analysis. As a result, lower limb ulcer showed a significant association with peritonitis in univariate and multivariate logistic regression models. The other factors such as DM were not significant in univariate logistic regression models. These results may indicate that the lower limb ulcer has an association with peritonitis in patients on PD; the patients with lower limb ulcer may develop to peritonitis earlier and more frequently than the patients without lower limb ulcer.

In contrast, Fisher's exact test analysis showed no significant difference in the presence of DM in PD patients with and without lower limb ulcer; univariate logistic regression analysis also showed no association with peritonitis. Moreover, the presence of DM was not significant in multivariate logistic regression analysis either; we thought presence of DM was considered as the most important confounding factor, because DM was one of the common problems of peritonitis and lower limb ulcer. Therefore, lower limb ulcer may have an association for peritonitis in patients on PD independent of DM. However, those results may be attributable to various factors because this study has certain limitations.

We investigate the patients on PD with lower limb ulcer in this study to analyze the association between lower limb ulcer and PD-related peritonitis in this study. 
Table 3 Logistic Regression Analysis for Peritonitis

\begin{tabular}{|c|c|c|c|c|}
\hline \multicolumn{5}{|c|}{ Logistic Regression Analysis for Peritonitis } \\
\hline \multirow[t]{2}{*}{ Variables } & \multicolumn{2}{|l|}{ Univariate Analysis } & \multicolumn{2}{|l|}{ Multivariate Analysis } \\
\hline & Odds Ratio ( $95 \% \mathrm{Cl})$ & $P$ value & Odds Ratio $(95 \% \mathrm{Cl})$ & $P$ value \\
\hline Exit-site infection & $1.055(0.219-3.908)$ & 0.941 & & \\
\hline Diabetes mellitus & $2.600(0.90 I-8.220)$ & 0.078 & $2.150(0.697-7.084)$ & 0.183 \\
\hline CVD at PD initiation & $1.417(0.439-4.240)$ & 0.546 & & \\
\hline Lower limb ulcer & $8.461(1.854-45.60)$ & 0.006 & $7.169(1.519-39.480)$ & 0.013 \\
\hline Abnormal status of $\mathrm{ABI}$ & $2.242(0.270-15.264)$ & 0.421 & & \\
\hline Smoking & $\mathrm{I} .450(0.50 \mathrm{I}-4.580)$ & 0.500 & & \\
\hline Diverticulosis & $1.760(0.612-5.082)$ & 0.291 & & \\
\hline Arrhythmia & $0.449(0.023-2.700)$ & 0.424 & & \\
\hline Icodextrin & $1.724(0.607-5.036)$ & 0.305 & & \\
\hline BMI $(\mathrm{kg} / \mathrm{m} 2)$ & $1.007(0.884-1.172)$ & 0.922 & & \\
\hline
\end{tabular}

Abbreviations; $\mathrm{Cl}$, confidence interval; CVD, cardiovascular disease; PD, peritoneal dialysis; $\mathrm{ABI}$, ankle-brachial pressure index; smoking, current and former smoking; $\mathrm{BMI}$, body mass index.

We attempted to identify the bacteria responsible for peritonitis and lower limb ulcer in our study subjects. We detected the same organ from cultures of lower limb ulcer and effluent at peritonitis in only one of the five patients who developed lower limb ulcer and peritonitis; in this one patient, we could not detect the organ from blood cultures. Therefore, the peritonitis may not have been caused by an infection of the bloodstream from the lower limb ulcer, but via touch contamination. In the other four patients, the responsible organs for peritonitis were different, based on the culture of their lower limb ulcer, or the organ from the culture of their lower limb ulcer was not detected.

Although we could not prove a direct association between peritonitis and lower limb ulcer in our patients, there might be some associations between peritonitis and lower limb ulcer in patients on PD, such as a problem in the immune system that may cause peritonitis and lower limb ulcer; both peritonitis and lower limb ulcer have mutual problems of infection. Moreover, peritonitis developed after lower limb ulcer in all five patients in this study. Thus, we might have to manage patients undergoing PD with lower limb ulcer more carefully because they might be at a higher risk of PD-related peritonitis.

Although this was not the primary aim of our study, the icodextrin and BMI were also significantly different between the patients with and without lower limb ulcer. All patients with lower limb ulcer have the episode of using dialysate including icodextrin for ultrafiltration failure. Lower limb edema is common problem in patients on PD. Especially, lower limb edema was more common and severe in the patients with ultrafiltration failure. Lower limb edema has bad effect for lower limb ulcer. BMI has also significant difference between the patients with and without lower limb ulcer in unpaired $t$-test analysis. Therefore, icodextrin and higher BMI may have some associations with lower limb ulcer in patients on PD, although we could not prove the clear associations in these results in this study because of some limitations.

This study has several limitations. First, the number of participants was relatively small and was not enough to allow robust statistical analyses because this study was conducted at a single center, and the total observation period of this study was relatively short ( 5 years) and the follow-up period of patients was also short. Second, the number of patients with lower limb ulcer and peritonitis was small. Third, this study was not an interventional study, but a retrospective observational study; therefore, the results may contain some bias. Forth, the period occurred lower limb ulcer of the patients were different, not at the PD initiation and the initiation of this study.

Therefore, further large-scale, prospective studies are required to confirm the results of this study.

\section{Conclusion}

In conclusion, lower limb ulcer may be associated with peritonitis in patients undergoing PD. Further large-scale, prospective studies are required to confirm these results.

\section{Acknowledgments}

The authors thank all the participants and the staff of Nippon Medical School Hospital Blood Purification Unit. 


\section{Disclosure}

The authors report no conflicts of interest in this work.

\section{References}

1. Li PK, Szeto CC, Piraino B, et al. ISPD peritonitis recommendations: 2016 update on prevention and treatment. Perit Dial Int. 2016;36 (5):481-508. doi:10.3747/pdi.2016.00078

2. Mehrotra R, Devuyst O, Davies SJ, Johnson DW. The current state of peritoneal dialysis. J Am Soc Nephrol. 2016;27(11):3238-3252. doi:10.1681/ASN.2016010112

3. Ye H, Zhou Q, Fan L, et al. The impact of peritoneal dialysis-related peritonitis on mortality in peritoneal dialysis patients. BMC Nephrol. 2017;18(1):186. doi:10.1186/s12882-017-0588-4

4. Cho Y, Johnson DW. Peritoneal dialysis-related peritonitis: towards improving evidence, practices, and outcomes. Am J Kidney Dis. 2014;64(2):278-289. doi:10.1053/j.ajkd.2014.02.025

5. McDonald SP, Collins JF, Rumpsfeld M, Johnson DW. Obesity is a risk factor for peritonitis in the Australian and New Zealand peritoneal dialysis patient populations. Perit Dial Int. 2004;24 (4):340-346. doi:10.1177/089686080402400408

6. See EJ, Johnson DW, Hawley CM, et al. Early peritonitis and its outcome in incident peritoneal dialysis patients. Perit Dial Int. 2017. doi:10.3747/pdi.2016.00158

7. Bolton L. Preventing peritoneal dialysis infections. Wounds. 2019;31 (6):163-165.

8. Yip T, Tse KC, Lam MF, et al. Colonic diverticulosis as a risk factor for peritonitis in Chinese peritoneal dialysis patients. Perit Dial Int. 2010;30(2):187-191. doi:10.3747/pdi.2007.00244

9. Tsai CC, Lee JJ, Liu TP, et al. Effects of age and diabetes mellitus on clinical outcomes in patients with peritoneal dialysis-related peritonitis. Surg Infect (Larchmt). 2013;14(6):540-546. doi:10.1089/ sur.2012.195

10. Benabed A, Bechade C, Ficheux M, Verger C, Lobbedez T. Effect of assistance on peritonitis risk in diabetic patients treated by peritoneal dialysis: report from the French language peritoneal dialysis registry. Nephrol Dial Transplant. 2016;31(4):656-662. doi:10.1093/ndt/ gfw011

11. Kosmadakis G, Albaret J, Da Costa Correia E, Somda F, Aguilera D. Constipation in peritoneal dialysis patients. Perit Dial Int. 2019;39 (5):399-404. doi:10.3747/pdi.2018.00169

12. United States Renal Data System. 2019 USRDS Annual Data Report: Epidemiology of Kidney Disease in the United States. National Institutes of Health, National Institute of Diabetes and Digestive and Kidney Diseases; 2019.
13. Nitta K, Masakane I, Hanafusa N. Annual dialysis data report 2017, JSDT renal data registry. Ren Replace Ther. 2019;5:53. doi:10.1186/ s41100-019-0248-1

14. Kwong VW, Li PK. Peritoneal dialysis in Asia. Kidney Dis. 2015;1 (3):147-156. doi:10.1159/000439193

15. Andrulli S, Chiavenna C, Bigi MC, et al. Predictors of first ischemic lower limb ulcer in dialysis patients: an observational cohort study. J Nephrol. 2018;31(3):435-443. doi:10.1007/s40620-017-0429-3

16. Wang IK, Hsu $\mathrm{CH}$, Tsai $\mathrm{CH}$, et al. Comparison of the risk of non-traumatic lower extremity amputation between haemodialysis and peritoneal dialysis patients with end-stage renal disease. Nephrology. 2018;23(1):86-92. doi:10.1111/nep.12946

17. Kaminski MR, Lambert KA, Raspovic A, et al. Risk factors for foot ulceration in adults with end-stage renal disease on dialysis: a prospective observational cohort study. BMC Nephrol. 2019;20 (1):423. doi:10.1186/s12882-019-1594-5

18. Bourrier M, Ferguson TW, Embil JM, Rigatto C, Komenda P, Tangri N. Peripheral artery disease: its adverse consequences with and without CKD. Am J Kidney Dis. 2020;75(5):705-712. doi:10.1053/j.ajkd.2019.08.028

19. Kaminski MR, Raspovic A, McMahon LP, et al. Factors associated with foot ulceration and amputation in adults on dialysis: a cross-sectional observational study. BMC Nephrol. 2017;18(1):293. doi:10.1186/s12882-017-0711-6

20. Kaminski MR, Raspovic A, McMahon LP, Erbas B, Landorf KB. Risk factors for foot ulceration in adults with end-stage renal disease on dialysis: study protocol for a prospective observational cohort study. J Foot Ankle Res. 2015;8(1):53. doi:10.1186/s13047-0150110-9

21. Allison GM, Flanagin E. How ESKD complicates the management of diabetic foot ulcers: the vital role of the dialysis team in prevention, early detection, and support of multidisciplinary treatment to reduce lower extremity amputations. Semin Dial. 2020;33(3):245-253. doi:10.1111/sdi.12875

22. Wukich DK, Raspovic KM, Suder NC. Patients with diabetic foot disease fear major lower-extremity amputation more than death. Foot Ankle Spec. 2018;11(1):17-21. doi:10.1177/1938640017694722

23. Lavery LA, Hunt NA, Ndip A, Lavery DC, Van Houtum W, Boulton AJ. Impact of chronic kidney disease on survival after amputation in individuals with diabetes. Diabetes Care. 2010;33 (11):2365-2369. doi:10.2337/dc10-1213

24. Pliakogiannis T, Bailey S, Cherukuri S, et al. Vascular complications of the lower extremities in diabetic patients on peritoneal dialysis. Clin Nephrol. 2008;69(5):361-367. doi:10.5414/CNP69361
The International Journal of Nephrology and Renovascular Disease is an international, peer-reviewed open-access journal focusing on the pathophysiology of the kidney and vascular supply. Epidemiology, screening, diagnosis, and treatment interventions are covered as well as basic science, biochemical and immunological studies. The manuscript management system is completely online and includes a very quick and fair peer-review system, which is all easy to use. Visit http://www.dovepress.com/testimonials.php to read real quotes from published authors. 